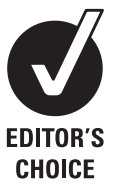

CHOICE
Australian Research Centre in Sex, Health and Society, La Trobe University, Melbourne Australia

\section{Correspondence to} Dr Anthony Lyons, Australian Research Centre in Sex, Health and Society, La Trobe University, 215 Franklin Street, Melbourne, Victoria 3000

Australia;

a.lyons@latrobe.edu.au

Accepted 10 December 2011 Published Online First 12 January 2012

\title{
Age at first anal sex and HIV/STI vulnerability among gay men in Australia
}

\author{
Anthony Lyons, Marian Pitts, Jeffrey Grierson, Anthony Smith, Stephen McNally, \\ Murray Couch
}

\begin{abstract}
Objectives To determine whether there is a link between age at first anal intercourse (AFAl) and gay men's HIV/ sexually transmissible infection (STI) vulnerability, including tendencies to engage in higher risk sexual behaviour.
\end{abstract}

Methods A nationwide cross-sectional survey was conducted online involving 845 Australian gay men born between 1944 and 1993.

Results Median AFAl fell from 35 years for men born between 1944 and 1953 to 18 years for men born between 1984 and 1993. Of those who reported having had anal intercourse ( $N=822$ ), HIV-positive men were found to be significantly younger on average when they first had anal intercourse compared with HIV-negative men (18.5 vs 21.3 years, $\mathrm{p}<0.001)$. Men with a history of other STIs were also significantly younger. Engaging in higher risk sexual behaviour is a likely factor, with AFAl generally younger among men who reported $>10$ sexual partners in the past year $(p<0.001)$ and who engaged in group sex $(p<0.001)$, receptive anal intercourse $(p=0.008)$ or were drug or alcohol affected $(p=0.06)$ during their most recent sexual encounter.

Conclusions There appears to be a strong link between AFAl and infection with HIV/STIs, as well as tendencies to engage in higher risk sexual behaviour. While further research is needed to understand this link, these findings highlight a need for sexuality education aimed at gayidentified youth to ensure their sexual debut does not lead to poorer sexual health outcomes.

\section{INTRODUCTION}

Gay men continue to carry a disproportionate burden of HIV. Worryingly, rates of new diagnoses have increased in almost all developed countries over the past decade, ${ }^{1}$ despite many of these countries investing heavily in safer sex programmes. In light of these trends, new information may be needed about factors associated with HIV infection and higher risk sexual behaviour. The age at which men first have anal intercourse (AFAI) with another man may be one important factor. For instance, many young samesex attracted adolescents have sex, yet receive no homosexual-based sex education from parents, peers or schools, ${ }^{2}{ }^{3}$ potentially leaving them with limited understanding of safer sex practices. This is particularly concerning, given that our earliest sexual experiences often begin a pattern that persists in future years, such as not using condoms. ${ }^{4} 5$ Thus, in the absence of adequate education, engaging in anal intercourse at a young age may often be unprotected and may establish patterns involving riskier behaviour that extend into the future. In fact, a recent study found the earlier that gay men had their first experience of anal intercourse, the less likely they were to have used a condom during that experience. ${ }^{6}$ For some, having anal intercourse at an early age may also indicate sexual adventurism, ${ }^{7}$ thus also providing a marker for future higher risk behaviour.

At present, the only indications of links between AFAI and future HIV or higher risk sexual behaviour among gay men come from research involving heterosexual men and women in which age at first intercourse has been shown to be a strong marker for future sexual health and behaviour patterns. For example, compared with adults who had their first intercourse later, those who had their debut in early to mid-adolescence tend to have a greater number of sexual partners, ${ }^{8-11}$ are more likely to engage in higher risk sexual practices, ${ }^{8}{ }^{10-12}$ have a higher prevalence of sexually transmissible infections (STIs), ${ }^{811}$ as well as a higher prevalence of sexual difficulties or dysfunctions, ${ }^{8}$ and tend to have more negative attitudes towards condom use. ${ }^{8}$ These findings are particularly concerning due to continued declines in the median age of first sexual intercourse, ${ }^{13}$ now under 18 years for both heterosexual men and women ${ }^{8} 14$ and gay $\operatorname{men}^{2} 6$ in a number of countries worldwide.

While detailed information about the association between age at first intercourse for heterosexual men and women and their future sexual health is already guiding sex education and other sexual health strategies, ${ }^{15-17}$ a need exists for similar information on gay men. Using data collected from a nationwide cross-sectional online survey of Australian gay men born between 1944 and 1993 (aged 16-65 years), we report (1) patterns of AFAI across five age cohorts, (2) socio-demographic differences in AFAI and (3) associations between AFAI and future HIV infection, other STIs and sexual behaviour.

\section{METHODS \\ Study population}

The survey was completed online by 1069 men. These men were all born before 1994 and were from every state and territory in Australia, including major cities, regional towns and rural areas. From this initial sample, we made the following exclusions: men who identified as non-homosexual (to focus on gay men) and men born before 1944 (due to 
only nine men in this group). This resulted in a total sample of 845 men born between 1944 and 1993 (aged 16-65 years) and who identified as homosexual. This study was undertaken with approval from the La Trobe University Human Ethics Committee.

\section{Survey}

Data in this study came from a larger online survey in which men born before 1994 retrospectively reported on their experiences involving sex and relationships. In the part of the survey presented in this paper, men were asked a range of sociodemographic questions, including their age, country of birth, country of residence, education level, employment status and whether they were in a regular relationship. They then reported on their sexual behaviour. For this paper, we focused on potentially higher risk behaviours with regard to HIV/STI infection. Men were asked whether they had ever had anal intercourse and the age at which they first had anal intercourse. They reported the number of sexual partners they had during the past 12 months before describing their most recent encounter, including the number of partners, type of partners (casual, occasional, regular), whether they had insertive or receptive anal intercourse, used condoms or were affected by drugs or alcohol. In the last part of the survey, men reported on their sexual health, including HIV testing, their HIV status, number of STIs in the past 12 months (genital herpes, thrush, chlamydia, genital warts, gonorrhoea, pubic lice, syphilis, non-specific urethritis) and any hepatitis $\mathrm{A}, \mathrm{B}$ or $\mathrm{C}$ diagnoses.

\section{Data collection}

The study was conducted between September 2008 and March 2009. Advertisements were placed on a variety of websites. Some of these were services for men looking for sexual partners and others were community-based news and information sites, such as HIV/AIDS organisations or gay community groups. Advertisements were also placed in the print media, such as gay community news and information magazines. All advertisements directed men to the online survey, where they first read some background information about the study, including assurances that their responses would be confidential and anonymous. Men were asked to confirm they had read this information before starting the survey. Completing the survey took approximately $20 \mathrm{~min}$. No identifying information was collected. Participation was entirely voluntary; no reward or compensation was offered.

\section{Data analysis}

Sample characteristics were compiled using descriptive statistics. We explored AFAI across five age cohorts using Kaplan-Meier survival curves. This technique estimates median age of AFAI and presents the cumulative proportion that report anal intercourse by a particular age. It is commonly used in studies of sexual debut, including those with cross-sectional data ${ }^{14} 18$ and takes into account the possibility that some individuals may not yet have had anal intercourse. All remaining analyses were restricted to those who reported having had anal intercourse and the age at which they had anal intercourse and used standard linear regression in which AFAI was the dependent variable. To avoid arbitrary cut-offs for age at first anal intercourse, we analysed AFAI as a continuous variable with the primary aim of identifying significant links between the age at which men first had anal intercourse and a range of sexual health and behaviour variables. Preliminary inspections of the data for each of the main independent variables showed AFAI to be distributed linearly. A multivariate linear regression involving all socio- demographic variables was first conducted to determine whether each variable was significantly associated with AFAI while simultaneously taking into account differences in the other variables. Separate linear regressions were then conducted for each sexual health and behaviour variable to again determine the degree to which these were associated with AFAI, while also controlling for those socio-demographic variables that were significantly associated with AFAI. All variables were treated as significant at $p<0.05$ based on Wald tests of the overall effect of each factor. Analyses were conducted using Stata V.11.1.

\section{RESULTS}

\section{Sample characteristics}

Table 1 displays the numbers and percentages of men according to a range of socio-demographic characteristics and their age at first anal intercourse. Almost all men in our sample reported having had anal intercourse, with only 14 (2\%) saying they had either never had sex or never had anal intercourse. Of the men who had anal intercourse and reported their AFAI ( $\mathrm{N}=822), 55$ $(7 \%)$ reported being forced or frightened into having sex before they were 16 years of age, which is the legal age of consent for sexual intercourse in most states and territories of Australia. ${ }^{19}$ Of these 55 men, 15 reported having their first anal intercourse during a forced sexual encounter prior to age 16 .

\section{Age at first anal intercourse}

Figure 1 displays Kaplan-Meier survival curves of AFAI for five age cohorts. As shown, increasingly larger proportions of each

Table 1 Sample characteristics $(N=845)$

\begin{tabular}{|c|c|}
\hline & $\mathbf{N}(\%)$ \\
\hline \multicolumn{2}{|l|}{ Age cohort } \\
\hline 1984-1993 (currently $16-25$ years) & $209(25)$ \\
\hline 1974-1983 (currently $26-35$ years) & $248(30)$ \\
\hline 1964-1973 (currently $36-45$ years) & $213(25)$ \\
\hline 1954-1963 (currently $46-55$ years) & $112(13)$ \\
\hline 1944-1953 (currently 56-65 years) & $56(7)$ \\
\hline \multicolumn{2}{|l|}{ Education } \\
\hline Secondary or below & $231(27)$ \\
\hline Post-secondary & $610(73)$ \\
\hline \multicolumn{2}{|l|}{ Employment status } \\
\hline Unemployed & $109(13)$ \\
\hline Part time & $141(17)$ \\
\hline Full time & $593(70)$ \\
\hline \multicolumn{2}{|l|}{ Country of birth } \\
\hline Australia & $684(81)$ \\
\hline Overseas & $158(19)$ \\
\hline \multicolumn{2}{|l|}{ Relationship status } \\
\hline In an ongoing relationship & 359 (43) \\
\hline Not in an ongoing relationship & $484(57)$ \\
\hline \multicolumn{2}{|l|}{ Sexual coercion prior to age 16} \\
\hline No & $784(93)$ \\
\hline Yes & $55(7)$ \\
\hline \multicolumn{2}{|l|}{ Ever had anal intercourse } \\
\hline No & $14(2)$ \\
\hline Yes & $831(98)$ \\
\hline \multicolumn{2}{|l|}{ Age at first anal intercourse ${ }^{*}$} \\
\hline 15 years or younger & $118(14)$ \\
\hline $16-19$ years & $315(38)$ \\
\hline $20-24$ years & $212(26)$ \\
\hline 25 years or older & $177(21)$ \\
\hline
\end{tabular}


cohort reported engaging in anal intercourse in their adolescent years. Median AFAI was substantially older for men born between 1944 and 1953 (35 years) than for those in subsequent cohorts (1954-1963: 22 years; 1964-1973: 21 years; 1974-1983: 20 years; 1984-1993: 18 years).

All further analyses presented in this paper were restricted to men who reported ever having had anal intercourse and who reported their AFAI $(\mathrm{N}=822)$. As stated earlier, only 14 of 845 men $(2 \%)$ reported never having had anal intercourse and a further nine reported having had anal intercourse but not the age at which this first occurred. A multivariate linear regression was conducted to determine whether a range of socio-demographic variables (age cohort, education, employment status, country of birth, relationship status and sexual coercion prior to age 16) were each significantly associated with AFAI while taking into account differences in the other variables. As shown in table 2 , education $(\mathrm{F}[1,792]=10.91, \mathrm{p}=0.001)$ and a history of sexual coercion prior to age $16(\mathrm{~F}[1,792]=26.46, \mathrm{p}<0.001)$ were significantly associated with AFAI. In particular, men with a primary or secondary education as their highest attainment or who reported a history of sexual coercion prior to age 16 were younger on average when they first had anal intercourse. Not unexpectedly, AFAI also varied significantly by age cohort (ie, the five cohorts in which men were born; $F[4,792]=38.43$, $p<0.001)$. However, given that patterns for these cohorts closely followed those of the medians reported in the Kaplan-Meier analysis above, we decided not to report means in table 2 to avoid confusion between the two analyses.

\section{Age at first anal intercourse and sexual behaviour}

Table 3 displays mean AFAI for a range of variables that describe sexual behaviour over the past 12 months and in the men's most recent sexual encounter. Separate linear regressions were conducted for each variable. Because age cohort, education and sexual coercion prior to age 16 were significantly associated with AFAI, all models were adjusted for differences in these variables. As shown in table 3 , the numbers of sexual partners that men reported having in the past 12 months were significantly associated with AFAI $(\mathrm{F}[3,793]=7.06, \mathrm{p}<0.001)$. In particular, men with $>10$ partners were younger on average when they first had anal intercourse compared with those who reported fewer partners but were no younger than the few men who reported having had no partners at all.

With regard to the men's most recent sexual encounter, the number of partners involved $(\mathrm{F}[1,788]=21.49, \mathrm{p}<0.001)$ and the position taken for anal intercourse $(F[3,786]=3.93, p=0.008)$

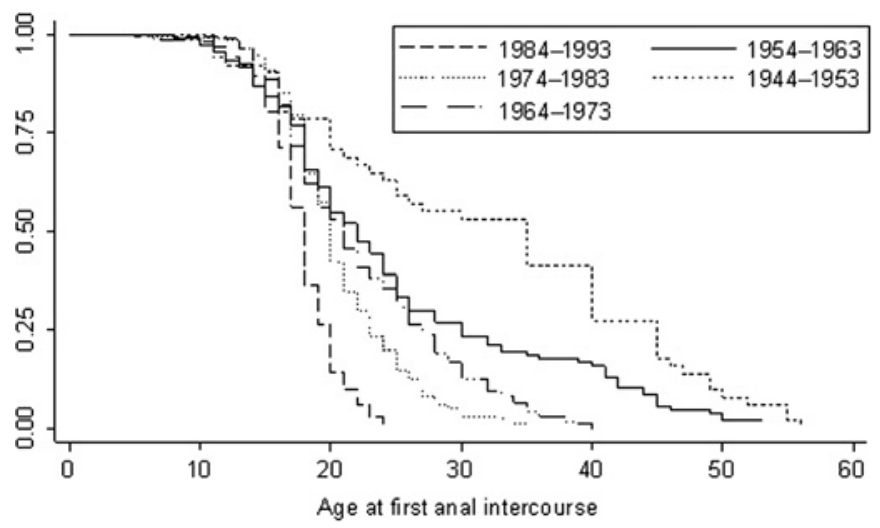

Figure 1 Kaplan-Meier survival curves for age at first anal intercourse according to men's age cohort.
Table 2 Mean age at first anal intercourse according to sample characteristics* $^{*}$

\begin{tabular}{lllll}
\hline & Mean AFAI & $\boldsymbol{\beta}$ & $\mathbf{t}$ & $\mathbf{p ~ V a l u e}$ \\
\hline Education & & & & \\
$\quad$ Post-secondary $\dagger$ & 21.5 & - & - & - \\
$\quad$ Secondary or below & 19.4 & -0.11 & 3.30 & 0.001 \\
Employment & & & & \\
$\quad$ Full time $\dagger$ & 20.9 & - & - & - \\
$\quad$ Part time & 19.7 & -0.04 & 1.18 & 0.24 \\
$\quad$ Unemployed & 22.2 & 0.01 & 0.21 & 0.83 \\
Country of birth & & & & \\
$\quad$ Australia $\dagger$ & 20.7 & - & - & - \\
$\quad$ Overseas & 21.8 & 0.02 & 0.70 & 0.48 \\
Relationship status & & & & \\
$\quad$ Not in an ongoing relationship $\dagger$ & 20.9 & - & - & - \\
$\quad$ In an ongoing relationship & 20.9 & -0.02 & 0.51 & 0.61 \\
Sexual coercion prior to age 16 & & & & \\
$\quad$ No $\dagger$ & 21.2 & - & - & - \\
Yes & 16.4 & -0.16 & 5.14 & $<0.001$ \\
\hline
\end{tabular}

* Sample is restricted to those who reported ever having had anal intercourse and who reported the age at which they first had anal intercourse $(\mathrm{N}=822)$.

†Reference category.

AFAl, age at first anal intercourse; $\beta$, standardised beta coefficient; $t, t$ test.

were both significantly associated with AFAI. Whether men were affected by drugs or alcohol was marginally significant $(\mathrm{F}$ $[1,775]=3.58, p=0.06)$. In summary, men who had group sex, engaged in receptive anal intercourse or were affected by drugs or alcohol were significantly younger on average when they first had anal intercourse compared with other men. Use of condoms and having sex with a casual or occasional partner were not significantly associated with AFAI.

All analyses above controlled for the age cohort in which men were born. Because these cohorts were categorical, we conducted the analyses a second time using age as a continuous variable to check whether the same findings emerged. Findings were almost identical. No differences were found with regard to levels of statistical significance, including analyses involving men affected by drugs or alcohol, which remained marginally significant $(\mathrm{p}=0.07)$.

\section{Age at first anal intercourse and HIV/STls}

Table 3 also displays mean AFAI for a range of variables that describe men's HIV/STI history. Following the same procedure above, linear regressions were conducted for each variable while controlling for age cohort, education and sexual coercion prior to age 16. As shown in table 3, HIV status $(F[1,654]=16.46$, $\mathrm{p}<0.001)$, number of STIs in the past 12 months $(\mathrm{F}[2,799]=3.79$, $\mathrm{p}=0.02)$ and a history of hepatitis $\mathrm{A}(\mathrm{F}[1,782]=19.10, \mathrm{p}<0.001)$, hepatitis $B(F[1,784]=25.69, p<0.001)$ and hepatitis $C(F$ $[1,784]=14.60, p<0.001)$ were all significantly associated with AFAI. In summary, men who were HIV positive, reported multiple STIs in the past 12 months and reported ever receiving a diagnosis for hepatitis $\mathrm{A}, \mathrm{B}$ or $\mathrm{C}$ were significantly younger on average when they first had anal intercourse than other men. Those who reported never having had an HIV test also tended to be younger, but after controlling for age and other socio-demographics, this association was not quite significant $(\mathrm{F}[1,795]=$ 3.18, $\mathrm{p}=0.07$ ).

To determine whether these patterns were the same for all five age cohorts, a series of linear regressions were conducted to test for significant interactions between the cohorts and each sexual health variable. These regressions also controlled for education and sexual coercion prior to age 16. All interactions were 
Table 3 Mean age at first anal intercourse according to current sexual behaviour and HIV/STIs

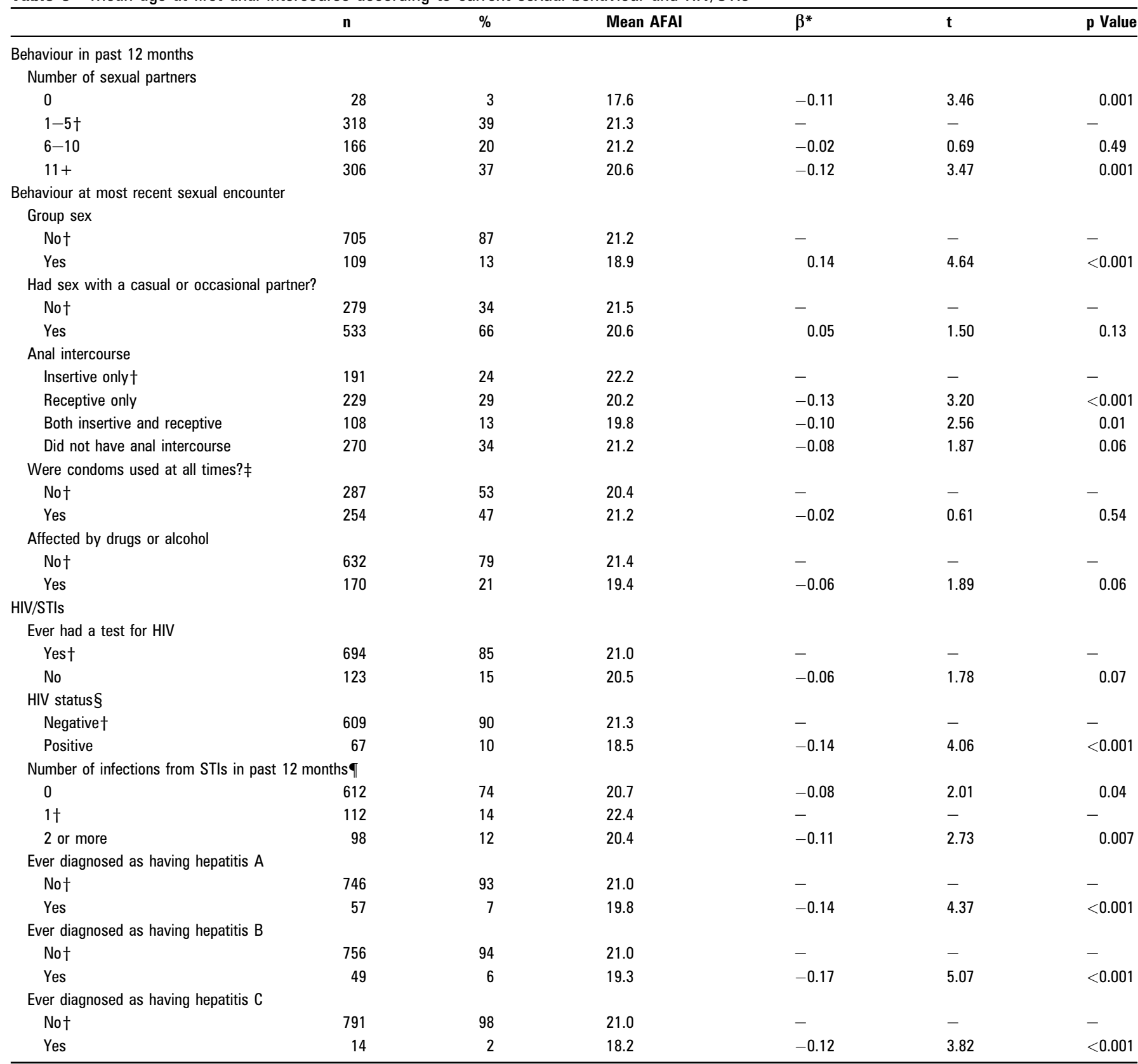

$p$ Values relate to differences in mean AFAI between each category of a variable and its reference category.

${ }^{*}$ Adjusted for age cohort, education and sexual coercion prior to age 16 years.

†Reference category.

$\ddagger$ Of those who had anal intercourse at their most recent sexual encounter $(\mathrm{N}=543)$.

§Of men who reported having had an HIV test $(\mathrm{N}=694)$.

ๆIncludes genital herpes, thrush, chlamydia, genital warts, gonorrhoea, pubic lice, syphilis and non-specific urethritis.

AFAl, age at first anal intercourse; $\beta$, standardised beta coefficient; STls, sexually transmissible infections; $t$, $t$ test.

non-significant, which suggests that patterns between AFAI and HIV/STI history are broadly similar across the cohorts. For example, men who reported being HIV positive were younger on average when they first had anal intercourse than HIV-negative men for all five cohorts (1984-1993: 16.7 vs 17.5 years; 1974-1983: 18.5 vs 19.9 years; $1964-1973$ : 17.1 vs 22.4 years; 1954-1963: 20.1 vs 23.5 years and 1944-1953: 26.6 vs 29.2 years).

In addition, for the same reasons as described in the section on sexual behaviour, all analyses that involved HIV/STIs were performed a second time using age as a continuous variable. Findings reported were almost identical to those that used age as a categorical variable. No differences were found with regard to levels of statistical significance, including analyses involving HIV testing, which remained marginally significant $(p=0.06)$.

\section{DISCUSSION}

To our knowledge, this is the first study that links AFAI with HIV/STIs and higher risk sexual behaviour among gay men. Based on the sample in this study, the age at which men first have anal intercourse appears to be a strong marker for HIV. Those who reported being HIV positive were younger on average when they first had anal intercourse. Importantly, this association remains strong even after controlling for 
socio-demographic differences in AFAI, including men's age, education or whether they were victims of childhood sexual abuse. Studies linking HIV with sexual debut among Westerners are rare. ${ }^{20}$ However, our findings are consistent with those that show heterosexual men and women with the highest incidence of STIs tend to report early age of first intercourse. ${ }^{21}$ Just recently, a US study also reported links between age at sexual debut and some higher risk sexual behaviours among ethnic minority young men who have sex with men. ${ }^{22}$

One factor likely to contribute to this vulnerability is the association we found between AFAI and recent experiences of higher risk sexual behaviour, including having a large number of sexual partners in the past 12 months, engaging in group sex and having receptive anal intercourse. Although no differences were found for condom use, engaging in unprotected sex with casual partners is not uncommon among gay men, ${ }^{23}$ which is obviously made riskier if they also have multiple partners involving receptive anal intercourse. Early sexual debut and a tendency towards having numerous sexual partners may be signs of sexual adventurism or sensation seeking. ${ }^{24}$ However, our findings around receptive anal intercourse are particularly intriguing. One explanation may relate to recent findings that suggest our earliest sexual experiences can set a pattern for future sexual behaviour. ${ }^{4} 5$ For example, adopting the receptive role is common among younger gay men, ${ }^{25}$ possibly because many of these men tend to have older partners who take an insertive role. ${ }^{626}$ For some, the role they take plays a major part in their sexual identity where they view themselves as either tops or bottoms. ${ }^{27}$ Consequently, if their earliest sexual experiences primarily involve receptive anal intercourse, some of these men may form identities around being bottoms and continue favouring receptive over insertive anal intercourse.

AFAI was also associated with other aspects of sexual health beyond HIV. While having an STI in the past year was not linked to AFAI, those who reported multiple STIs were younger on average when they first had anal intercourse than those who did not report an STI. Those with histories of hepatitis A, B and $C$ were also younger on average when they first had anal intercourse. With all these links between gay men's AFAI and their future sexual health, placing a stronger emphasis on addressing the needs of those who had their first anal intercourse at a particularly young age may need to be an important part of preventing HIV/STIs in this population. Understanding the life histories of these men and the circumstances surrounding their first sexual experiences may also provide clues about how to target safer sex education and other forms of support.

Given our findings, it may be tempting to assume that an effective HIV prevention strategy would involve promoting delayed sexual debut among gay men. However, our data provide no information about whether a causal link exists between AFAI and future sexual health, only that it is a strong marker. Also, existing efforts to promote delayed sexual debut among young people, particularly in the USA, appear to have had little success as HIV prevention strategies. ${ }^{15}$ Assuming many gay youth will continue having early sexual intercourse, it is particularly important for sexuality education in schools to teach strategies for safer sex among same-sex attracted men. While it is common for secondary schools in many countries to provide sexuality education, few programmes address issues around sex between gay men. This may explain recent US findings ${ }^{2}$ in which a large proportion of young gay men reported having little knowledge about HIV and STIs when they first had anal intercourse. Encouragingly, the few sexuality education programmes that have included topics relevant to gay men appear to result in less risky sexual behaviour, at least in the USA. $^{3}{ }^{28}$ Further efforts are clearly needed to incorporate sexuality education into school education for gay youths. However, in light of the strong association we found between AFAI and poorer education, these efforts will need to be conducted sufficiently early to catch those who dropout or leave school.

Another notable finding was the sharp decline in median AFAI between men born 1944-1953 and subsequent age cohorts. Although it is highly likely that declines in AFAI are a result of changes in socio-cultural context, many of those born between 1944 and 1953 would have been sexually active during the earliest years of the HIV epidemic. This was a time when highly active antiretroviral therapy was unavailable. Some of those who were having anal intercourse at an early age may have become infected with HIV and therefore not survived, which may at least partly explain the very high median AFAI for this group.

There were some limitations to this study that need to be considered when interpreting our findings. First, because this study involved a self-selected sample, we cannot claim it is representative of all Australian gay men. That said, our sample appeared to be diverse, with all of the main socio-demographic categories well represented. This diversity may have kept sampling bias to a minimum. Additionally, almost all significant associations involving AFAI were highly significant, so alternative sampling methods may not alter these findings.

Second, this study involved an online retrospective survey. Especially for older men, recalling their AFAI may incur a degree of recall error. Nevertheless, the first experience of anal intercourse is likely to be a significant event for many men and therefore memorable. Recall biases are also likely to be randomly distributed, at least within age groups, and therefore unlikely to affect comparisons between groups of men based on their AFAI. It should also be acknowledged that one benefit of an online survey is that it guarantees anonymity, so respondents are perhaps more likely to answer honestly, which is not always the case in other survey modes involving questions about sexual behaviour. $^{29} 30$

Third, this survey targets men who had experience with sex or relationships. This is a particular issue for the 1984-1993 cohort as some may not yet have had sex. That said, recent data shows a majority of young people are now having sex before age $17,{ }^{8} 14$ including same-sex attracted adolescents, ${ }^{2} 6$ so future studies are likely to produce a similar median AFAI to that found in our study. Moreover, while this limitation points to a need for further research, it is unlikely to have affected the main findings of this paper. That is, of men who have had anal intercourse, those with poorer sexual health outcomes tend to report an earlier AFAI

\section{Key messages}

- Findings from this nationwide survey show strong links between age at first anal intercourse and gay men's HIV/STI vulnerability.

- Gay men with a history of HIV and STIs were significantly younger on average when they first had anal intercourse than other men.

- Gay men with a recent history of higher risk sexual behaviour were also younger on average when they first had anal intercourse than other men. 
In summary, findings from this nationwide online survey reveal strong links between age at first anal intercourse and future sexual health and behaviour. In the sample presented in this paper, those who became HIV positive, engaged in higher risk sexual behaviour and reported other poorer sexual health outcomes tended to have had their first anal intercourse at a younger age than other men. With the median age of sexual debut continuing to decline, clinicians and other health service providers, as well as researchers, need to pay particular attention to gay men's earliest sexual experiences. In particular, men who report having anal intercourse at a young age are likely to be at heightened HIV vulnerability and therefore require additional education and support for ensuring a healthier sex life. Further education around safer sex practices that specifically targets gayidentified youth may also be required to ensure their sexual debut does not lead to poorer sexual health outcomes.

Acknowledgements We wish to thank Geoffrey Smith at the Australian Research Centre in Sex, Health and Society for his assistance with setting up the survey and collecting data. We also wish to thank Professor Judy Simpson and Dr Patrick Kelly at the University of Sydney, who both provided expert statistical advice.

Competing interests None

Ethics approval Ethics approval was provided by La Trobe University Human Ethics Committee.

Contributors MP, JG, AS, SM and MC designed the study. MP and AS supervised the collection of data. AL analysed the data and wrote the manuscript. All authors contributed to the manuscript.

Provenance and peer review Not commissioned; externally peer reviewed.

\section{REFERENCES}

1. Grulich $\mathbf{A E}$, Kaldor JM. Trends in HIV incidence in homosexual men in developed countries. Sex Health 2008;5:113-18.

2. Kubicek K, Beyer WJ, Weiss G, et al. In the dark: young men's stories of sexua initiation in the absence of relevant sexual health information. Health Educ Behav 2010;37:243-63.

3. Blake SM, Ledsky R, Lehman T, et al. Preventing sexual risk behaviors among gay, lesbian, and bisexual adolescents: the benefits of gay-sensitive HIV instruction in schools. Am J Public Health 2001;91:940-6.

4. Shafii T, Stovel K, Davis R, et al. Is condom use habit forming? Condom use at sexual debut and subsequent condom use. Sex Transm Dis 2004;31:366-72.

5. Shafii T, Stovel K, Holmes K. Association between condom use at sexual debut and subsequent sexual trajectories: a longitudinal study using biomarkers. Am J Public Health 2007:97:1090-5.

6. Balthasar $\mathbf{H}$, Jeannin A, Dubois-Arber F. First anal intercourse and condom use among men who have sex with men in Switzerland. Arch Sex Behav 2009;38:1000-8.

7. Kippax S, Campbell D, Van de Ven P, et al. Cultures of sexual adventurism as markers of HIV seroconversion: a case control study in a cohort of Sydney gay men. AIDS Care 1998;10:677-88.

8. Sandfort TG, Orr M, Hirsch JS, et al. Long-term health correlates of timing of sexual debut: results from a national US study. Am J Public Health 2008:98:155-61.
9. Cavazos-Rehg PA, Spitznagel EL, Bucholz KK, et al. The relationship between alcohol problems and dependence, conduct problems and diagnosis, and number of sex partners in a sample of young adults. Alcohol Clin Exp Res 2007;31:2046-52.

10. O'Donnell BL, O'Donnell CR, Stueve A. Early sexual initiation and subsequent sexrelated risks among urban minority youth: the reach for health study. Fam Plann Perspect 2001;33:268-75.

11. Coker AL, Richter DL, Valois RF, et al. Correlates and consequences of early initiation of sexual intercourse. J Sch Health 1994;64:372-7.

12. Lohman BJ, Billings A. Protective and risk factors associated with adolescent boy's early sexual debut and risky sexual behaviors. J Youth Adolesc 2008;37:723-35.

13. Wellings $\mathbf{K}$, Collumbien M, Slaymaker $\mathrm{E}$, et al. Sexual behaviour in context: a globa perspective. Lancet 2006;368:1706-28.

14. Cavazos-Rehg PA, Krauss MJ, Spitznagel EL, et al. Age of sexual debut among US adolescents. Contraception 2009;80:158-62.

15. Underhill K, Montgomery P, Operario D. Sexual abstinence only programmes to prevent HIV infection in high income countries: systematic review. BMJ 2007;335:248.

16. Mueller TE, Gavin LE, Kulkarni A. The association between sex education and youth's engagement in sexual intercourse, age at first intercourse, and birth control use at first sex. J Adolesc Health 2008;42:89-96.

17. Blake SM, Simkin L, Ledsky $\mathrm{R}$, et al. Effects of a parent-child communications intervention on young adolescents' risk for early onset of sexual intercourse. Fam Plann Perspect 2001;33:52-61.

18. Babalola S. Perceived peer behavior and the timing of sexual debut in Rwanda: a survival analysis of youth data. J Youth Adolesc 2004;33:353-63.

19. Lamont A. Age of Consent Laws. Melbourne: National Child Protection Clearinghouse, 2010.

20. Dillon FR, De La Rosa M, Schwartz SJ, et al. US Latina age of sexual debut: long term associations and implications for HIV and drug abuse prevention. AIDS Care 2010;22:431-40.

21. Rissel CE, Richters J, Grulich AE, et al. Sex in Australia: first experiences of vaginal intercourse and oral sex among a representative sample of adults. Aust N Z J Public Health 2003;27:131-7.

22. Outlaw AY, Phillips G 2nd, Hightow-Weidman LB, et al. Age of MSM sexual debut and risk factors: results from a multisite study of racial/ethnic minority YMSM living with HIV. AIDS Patient Care STDS 2011;25(Suppl 1):S23-9.

23. Van De Ven $\mathbf{P}$, Rawstorne $\mathrm{P}$, Crawford $\mathrm{J}$, et al. Increasing proportions of Australian gay and homosexually active men engage in unprotected anal intercourse with regular and with casual partners. AIDS Care 2002;14:335-41.

24. Bancroft J, Janssen E, Strong D, et al. Sexual risk-taking in gay men: the relevance of sexual arousability, mood, and sensation seeking. Arch Sex Behav 2003;32:555-72.

25. Lyons A, Pitts M, Smith G, et al. Versatility and HIV vulnerability: investigating the proportion of Australian gay men having both insertive and receptive anal intercourse. J Sex Med 2011:8:2164-71.

26. Middlethon AL. Sexual debut and the risk of HIV in young gay men in Norway. In: Aggleton P, Davies P, Hart G, eds. AIDS: Activism and Alliances. Bristol, PA: Taylor and Francis, 1997

27. Wegesin DJ, Meyer-Bahlburg HF. Top/bottom self-label, anal sex practices, HIV risk and gender role identity in gay men in New York City. J Psychol Human Sex 2000:12:43-62.

28. Goodenow C, Netherland J, Szalacha L. AIDS-related risk among adolescent males who have sex with males, females, or both: evidence from a statewide survey. Am J Public Health 2002;92:203-10.

29. Smith A, Lyons A, Pitts M, et al. Assessing knowledge of human papillomavirus and collecting data on sexual behavior: computer assisted telephone versus face to face interviews. BMC Public Health 2009:9:429.

30. Copas AJ, Wellings $K$, Erens $B$, et al. The accuracy of reported sensitive sexual behaviour in Britain: exploring the extent of change 1990-2000. Sex Transm Infect 2002:78:26-30. 